\title{
Muon anomalous magnetic moment, $Z$ boson decays, and collider physics in multicharged particles
}

\author{
Takaaki Nomura ${ }^{1, *}$ and Hiroshi Okada ${ }^{2,3, \dagger}$ \\ ${ }^{1}$ School of Physics, Korea Institute for Advanced Study (KIAS), Seoul 02455, Republic of Korea \\ ${ }^{2}$ Asia Pacific Center for Theoretical Physics (APCTP)_Headquarters San 31, \\ Hyoja-dong, Nam-gu, Pohang 790-784, Republic of Korea \\ ${ }^{3}$ Department of Physics, Pohang University of Science and Technology, Pohang 37673, Republic of Korea
}

(Received 28 March 2019; published 27 January 2020)

\begin{abstract}
We analyze the allowed region of the muon anomalous magnetic moment (muon $g-2$ ) satisfying lepton flavor violations, $Z$ boson decays, and collider physics, in a framework of multicharged particles. Then we explore the typical size of the muon $g-2$ and discuss which mode dominantly affects the muon $g-2$.
\end{abstract}

DOI: 10.1103/PhysRevD.101.015021

\section{INTRODUCTION}

The muon anomalous magnetic moment (muon $g-2$ ) is one of the promising phenomenologies to confirm the new physics. Therefore, there is still a discrepancy between the standard model (SM) and new physics [1]

$$
\Delta a_{\mu}=(26.1 \pm 8) \times 10^{-10},
$$

where the $3.3 \sigma$ deviation from the SM prediction with a positive value; recent theoretical analysis further indicates a $3.7 \sigma$ deviation [2]. Furthermore, several upcoming experiments such as Fermilab E989 [3] and J-PARC E34 [4] will provide a result in a more precise manner. From a theoretical point of view, several mechanisms have been historically proposed through, e.g., gauge contributions [5-7], Yukawa contributions at the one-loop level [8], and Barr-Zee contributions [9] at the two-loop level. In particular, when one supposes the muon $g-2$ is related to the other phenomenologies such as neutrino masses and the dark matter candidate, Yukawa contributions at the oneloop level would likely be promising candidates [10-33]. In this case, one has to simultaneously satisfy several constraints of lepton flavor violations (LFVs) such as $\ell_{i} \rightarrow \ell_{j} \gamma, \ell_{i} \rightarrow \ell_{j} \ell_{k} \bar{\ell}_{\ell}(i, j, k, \ell=(e, \mu, \tau))$, and leptonflavor-conserving (-violating) $Z$ boson decays such as $Z \rightarrow \ell \bar{\ell}^{\prime}, Z \rightarrow \nu \bar{\nu}^{\prime}$ [34]. Particularly, $\ell_{\mu} \rightarrow \ell_{e} \gamma$ gives the most stringent constraint, and the current branching ratio

\footnotetext{
"nomura@kias.re.kr

†hiroshi.okada@apctp.org
}

Published by the American Physical Society under the terms of the Creative Commons Attribution 4.0 International license. Further distribution of this work must maintain attribution to the author(s) and the published article's title, journal citation, and DOI. Funded by SCOAP. should be less than $4.2 \times 10^{-13}$ [35], and its future bound will reach $6 \times 10^{-14}$ [36]. Also, $Z$ boson decays will be tested by a future experiment such as CEPC [37].

In this paper, we introduce several multicharged fields (bosons and fermions) with general $U(1)_{Y}$ hypercharges to get the positive muon $g-2$, and we estimate the allowed region to satisfy all constraints of the muon $g-2$, LFVs, and $Z$ boson decays. Also, we consider the constraint of collider physics, since multicharged fields are severely restricted by the Large Hadron Collider (LHC). We discuss the necessity of an extra charged scalar in order to make exotic charged leptons decay into the SM particles and decay chains of exotic charged particles. Then the signature of exotic charged particles are explored, and we consider an allowed scenario accommodating muon $g-2$ and collider constraints.

This paper is organized as follows. In Sec. II, we review the model and formulate the LFVs, muon $g-2, Z$ boson decays, and renormalization group for $g_{Y}$. In Sec. III, we estimate the allowed region for each $N$, comparing to collider physics. We conclude in Sec. IV.

\section{MODEL SETUP AND CONSTRAINTS WITH A COMMON PART}

In our setup of the model, we introduce an isospin doublet fermion $L_{a}^{\prime} \equiv\left[\psi_{a}^{-n}, \psi_{a}^{-n-1}\right]^{T}(a=1)$ for simplicity ${ }^{1}$ and a new boson $h^{+n}$ with $n \equiv \frac{N-1}{2}(N=3,5, \ldots)$, as shown in Table I. Notice here that $N$ is defined by odd number, where $N=1$ is not considered because $L_{L}^{\prime}$ cannot be discriminated from $L_{L}$. The valid Lagrangian is given by

\footnotetext{
${ }^{1}$ When $L^{\prime}$ provides a flavor structure of the neutrino mass matrix, we minimally need two families to satisfy the neutrino oscillation data.
} 
TABLE I. Charge assignments of fields under $S U(2)_{L} \times U(1)_{Y}$, where $n \equiv \frac{N-1}{2}$ with $N=3,5, \ldots$, and all the new fields are color singlet.

\begin{tabular}{lccccc}
\hline \hline & $L_{L}$ & $e_{R}$ & $L_{L / R}^{\prime}$ & $H$ & $h^{+n}$ \\
\hline$S U(2)_{L}$ & $\mathbf{2}$ & $\mathbf{1}$ & $\mathbf{2}$ & $\mathbf{2}$ & $\mathbf{1}$ \\
$U(1)_{Y}$ & $-\frac{1}{2}$ & -1 & $-\frac{N}{2}$ & $\frac{1}{2}$ & $\frac{N-1}{2}$ \\
\hline \hline
\end{tabular}

$$
\begin{aligned}
-\mathcal{L}_{Y}^{n} & =f_{i a} \bar{L}_{L_{i}} L_{R_{a}}^{\prime} h^{n}+\text { H.c. } \\
& =f_{i a}\left[\bar{\nu}_{L_{i}} \psi_{a}^{-n} h^{n}+\bar{\ell}_{i} \psi_{a}^{-n-1} h^{n}\right]+\text { H.c. }
\end{aligned}
$$

where $i=1-3, a=1$ are generation indices. The Yukawa Lagrangian $y_{\ell_{i i}} \bar{L}_{L_{i}} e_{R_{i}} H$ provides masses for the charged leptons $\left(m_{\ell_{i}} \equiv y_{\ell_{i i}} v / \sqrt{2}\right.$ ) by developing a nonzero vacuum expectation value (VEV) of $H$, which is denoted by $\langle H\rangle \equiv v / \sqrt{2}$. The exotic lepton $L^{\prime}$ has vectorlike mass, and the new scalar field $h^{ \pm n}$ does not develop a VEV. We denote the mass of $L^{\prime}$ and $h^{ \pm n}$ by $m_{\psi}$ and $m_{h}$, respectively.

\section{A. Lepton flavor violations and the muon anomalous magnetic moment}

The Yukawa terms of $(f, g)$ give rise to $\ell_{i} \rightarrow \ell_{j} \gamma$ processes at the one-loop level. The branching ratio is given by

$$
B\left(\ell_{i} \rightarrow \ell_{j} \gamma\right) \approx \frac{48 \pi^{3} \alpha_{\mathrm{em}}}{G_{\mathrm{F}}^{2} m_{\ell_{i}}^{2}} C_{i j}\left(\left|a_{L_{i j}}\right|^{2}+\left|a_{R_{i j}}\right|^{2}\right),
$$

where $G_{\mathrm{F}} \approx 1.166 \times 10^{-5} \mathrm{GeV}^{-2}$ is the Fermi constant, $\alpha_{\mathrm{em}}\left(m_{Z}\right) \approx 1 / 128.9$ is the fine-structure constant [34], $C_{21} \approx 1$, $C_{31} \approx 0.1784$, and $C_{32} \approx 0.1736 . a_{L / R}$ is formulated as

$$
\begin{aligned}
a_{L_{i j}} \approx & -m_{\ell_{i}} \sum_{a=1-3} \frac{f_{j a} f_{a i}^{\dagger}}{(4 \pi)^{2}}\left[n F\left(\psi_{a}^{-n-1}, h^{n}\right)\right. \\
& \left.+(n+1) F\left(h^{n}, \psi_{a}^{-n-1}\right)\right], \\
a_{R_{i j}} \approx & -m_{\ell_{j}} \sum_{a=1-3} \frac{f_{j a} f_{a i}^{\dagger}}{(4 \pi)^{2}}\left[n F\left(\psi_{a}^{-n-1}, h^{n}\right)\right. \\
& \left.+(n+1) F\left(h^{n}, \psi_{a}^{-n-1}\right)\right],
\end{aligned}
$$

where $m_{\psi^{-n-1}} \equiv m_{\psi}$, and $m_{h^{n}} \equiv m_{h}$. The current experimental upper bounds are given by $[35,38]$

$$
\begin{aligned}
& B(\mu \rightarrow e \gamma) \leq 4.2 \times 10^{-13}\left(6 \times 10^{-14}\right), \\
& B(\tau \rightarrow \mu \gamma) \leq 4.4 \times 10^{-8}, \quad B(\tau \rightarrow e \gamma) \leq 3.3 \times 10^{-8},
\end{aligned}
$$

where in parentheses, $\mu \rightarrow e \gamma$ is a future reach of the MEG experiment [36].

\section{The muon anomalous magnetic moment $\left(\Delta a_{\mu}\right)$}

We can also estimate the muon anomalous magnetic moment through $a_{L, R}$, which is given by

$$
\Delta a_{\mu} \approx-m_{\mu}\left(a_{L}+a_{R}\right)_{22} .
$$

The $3.3 \sigma$ deviation from the $\mathrm{SM}$ prediction is $\Delta a_{\mu}=$ $(26.1 \pm 8) \times 10^{-10}[1]$ with a positive value.

\section{B. Flavor-conserving (-changing) leptonic $Z$ boson decays}

Here, we consider the $Z$ boson decay into two leptons through the Yukawa terms $f$ at one-loop level [23]. Since some components of $f$ are expected to be large so as to obtain a sizable $\Delta a_{\mu}$, the experimental bounds on $Z$ boson decays could be of concern at the one-loop level. First of all, the relevant Lagrangian is given $b^{2}$

\footnotetext{
${ }^{2}$ We neglect one-loop contributions in the SM.
}

$$
\begin{aligned}
\mathcal{L} \sim & \frac{g_{2}}{c_{w}}\left[\bar{\ell} \gamma^{\mu}\left(-\frac{1}{2} P_{L}+s_{W}^{2}\right) \ell+\frac{1}{2} \bar{\nu} \gamma^{\mu} P_{L} \nu\right] Z_{\mu} \\
& +\frac{g_{2}}{c_{w}}\left[\left(-\frac{1}{2} P_{L}+n s_{W}^{2}\right) \bar{\psi}^{n} \gamma^{\mu} \psi^{-n}\right. \\
& \left.+\left(-\frac{1}{2} P_{L}+(n+1) s_{W}^{2}\right) \bar{\psi}^{n+1} \gamma^{\mu} \psi^{-n-1}\right] Z_{\mu} \\
& +i n \frac{g_{2} s_{W}^{2}}{c_{W}}\left(h^{n} \partial^{\mu} h^{-n}-h^{-n} \partial^{\mu} h^{n}\right) Z_{\mu},
\end{aligned}
$$

where $s(c)_{W} \equiv \sin (\cos ) \theta_{W} \sim 0.23$ stands for the sine (cosine) of the Weinberg angle. The decay rate of the $\mathrm{SM}$ at tree level is then given by

$$
\begin{gathered}
\Gamma\left(Z \rightarrow \ell_{i}^{-} \ell_{j}^{+}\right)_{\mathrm{SM}} \approx \frac{m_{Z}}{12 \pi} \frac{g_{2}^{2}}{c_{W}^{2}}\left(s_{W}^{4}-\frac{s_{W}^{2}}{2}+\frac{1}{8}\right) \delta_{i j}, \\
\Gamma\left(Z \rightarrow \nu_{i} \bar{\nu}_{j}\right)_{\mathrm{SM}} \approx \frac{m_{Z}}{96 \pi} \frac{g_{2}^{2}}{c_{W}^{2}} \delta_{i j} .
\end{gathered}
$$

Combining all the diagrams in Fig. 1, the ultraviolet divergence cancels out, and only the finite part remains [23]. The resulting form is given by

$$
\begin{aligned}
& \Delta \Gamma\left(Z \rightarrow \ell_{i}^{-} \ell_{j}^{+}\right) \\
& \approx \frac{m_{Z}}{12 \pi} \frac{g_{2}^{2}}{c_{W}^{2}}\left[\frac{\left|B_{i j}^{\ell}\right|^{2}}{2}-\operatorname{Re}\left[A_{i j}\left(B^{\ell}\right)_{i j}^{*}\right]-\left(-\frac{s_{W}^{2}}{2}+\frac{1}{8}\right) \delta_{i j}\right],
\end{aligned}
$$



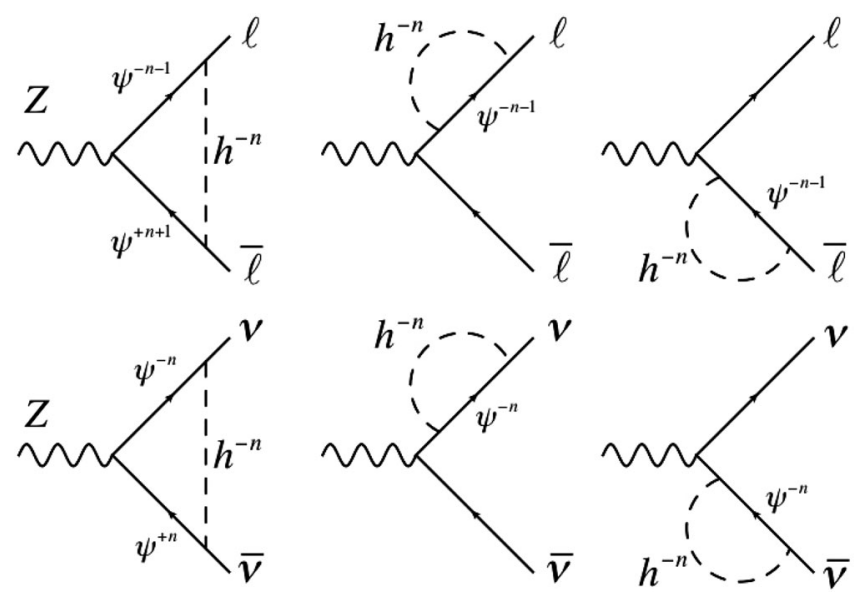

FIG. 1. Feynman diagrams for $Z \rightarrow \ell_{i} \bar{\ell}_{j}$ and $Z \rightarrow \nu_{i} \bar{\nu}_{j}$, where upper diagrams represent the contribution to $\bar{Z} \bar{\ell} \ell$, while the lower diagrams are for $Z \bar{\nu} \nu$.

$$
\Delta \Gamma\left(Z \rightarrow \nu_{i} \bar{\nu}_{j}\right) \approx \frac{m_{Z}}{24 \pi} \frac{g_{2}^{2}}{c_{W}^{2}}\left[\left|B_{i j}^{\nu}\right|^{2}-\frac{\delta_{i j}}{4}\right]
$$

where

$$
\begin{gathered}
A_{i j} \approx s_{W}^{2} \delta_{i j}, \quad B_{i j}^{\ell} \approx \frac{\delta_{i j}}{2}-\frac{f_{i a} f_{a j}^{\dagger}}{(4 \pi)^{2}} G^{\ell}(\psi, h) \\
B_{i j}^{\nu} \approx \frac{\delta_{i j}}{2}+\frac{f_{i a} f_{a j}^{\dagger}}{(4 \pi)^{2}} G^{\nu}(\psi, h) \\
G^{\ell}(\psi, h) \approx-n s_{W}^{2}\left(-\frac{1}{2}+s_{w}^{2}\right) H_{1}(\psi, h) \\
-\left(-\frac{1}{2}+s_{w}^{2}\right)^{2} H_{2}(\psi, h) \\
+\left(-\frac{1}{2}+(n+1) s_{w}^{2}\right) H_{3}(\psi, h) \\
G^{\nu}(\psi, h) \approx-n s_{W}^{2}\left(-\frac{1}{2}+s_{w}^{2}\right) H_{1}(\psi, h)-\frac{1}{2} H_{2}(\psi, h) \\
+\left(-\frac{1}{2}+n s_{w}^{2}\right) H_{3}(\psi, h), \\
H_{1}(1,2)=\frac{m_{1}^{4}-m_{2}^{4}+4 m_{1}^{2} m_{2}^{2} \ln \left[\frac{m_{2}}{m_{1}}\right]}{2\left(m_{1}^{2}-m_{2}^{2}\right)^{2}}
\end{gathered}
$$

$$
H_{3}(1,2)=m_{1}^{2}\left(\frac{m_{1}^{2}-m_{2}^{2}+2 m_{2}^{2} \ln \left[\frac{m_{2}}{m_{1}}\right]}{\left(m_{1}^{2}-m_{2}^{2}\right)^{2}}\right)
$$

Notice here that the upper index of $B$ represents $\psi \equiv \psi^{-n-1}$ for the charged-lepton final state, while $\psi \equiv \psi^{-n}$ for the neutrino final state. One finds the branching ratio by dividing the total $Z$ decay width $\Gamma_{Z}^{\text {tot }}=2.4952 \pm 0.0023 \mathrm{GeV}$ [34]. The current bounds on the lepton-flavor-changing (-conserving) $Z$ boson decay branching ratios at $95 \%$ C.L. are given by [34]

$$
\Delta \mathrm{BR}(Z \rightarrow \text { Invisible }) \approx \sum_{i, j=1-3} \Delta \mathrm{BR}\left(Z \rightarrow \nu_{i} \bar{\nu}_{j}\right)< \pm 5.5 \times 10^{-4}
$$

$$
\begin{aligned}
& \Delta \mathrm{BR}\left(Z \rightarrow e^{ \pm} e^{\mp}\right)< \pm 4.2 \times 10^{-5}, \\
& \Delta \mathrm{BR}\left(Z \rightarrow \mu^{ \pm} \mu^{\mp}\right)< \pm 6.6 \times 10^{-5}, \\
& \Delta \mathrm{BR}\left(Z \rightarrow \tau^{ \pm} \tau^{\mp}\right)< \pm 8.3 \times 10^{-5},
\end{aligned}
$$

$$
\begin{aligned}
& \operatorname{BR}\left(Z \rightarrow e^{ \pm} \mu^{\mp}\right)<7.5 \times 10^{-7}, \\
& \operatorname{BR}\left(Z \rightarrow e^{ \pm} \tau^{\mp}\right)<9.8 \times 10^{-6}, \\
& \operatorname{BR}\left(Z \rightarrow \mu^{ \pm} \tau^{\mp}\right)<1.2 \times 10^{-5},
\end{aligned}
$$

where $\Delta \operatorname{BR}\left(Z \rightarrow f_{i} \bar{f}_{j}\right)(i=j)$ is defined by

$\Delta \operatorname{BR}\left(Z \rightarrow f_{i} \bar{f}_{j}\right) \approx \frac{\Gamma\left(Z \rightarrow f_{i} \bar{f}_{j}\right)-\Gamma\left(Z \rightarrow f_{i} \bar{f}_{j}\right)_{\mathrm{SM}}}{\Gamma_{Z}^{\mathrm{tot}}}$.

We consider these constraints in our global analyses below.

\section{Beta function of $g_{Y}$}

Here we estimate the effective energy scale by evaluating the Landau pole for $g_{Y}$ in the presence of new exotic fields with nonzero multiple hypercharges. Each contribution of the new beta function of $g_{Y}$ from one $S U(2)_{L}$ doublet fermion with $-N / 2$ hypercharge is given by [19]

$$
\Delta b_{Y}^{f}=\frac{3}{5} \times \frac{4}{3} \times\left(\frac{N}{2}\right)^{2}
$$

Similarly, the contribution to the beta function from one $S U(2)_{L}$ singlet boson with $(N-1) / 2$ hypercharge is given by

$$
\Delta b_{Y}^{b}=\frac{3}{5} \times \frac{1}{3} \times\left(\frac{N-1}{2}\right)^{2}
$$

where $3 / 5$ is the rescaled coefficient. Then one finds the energy evolution of the gauge coupling $g_{Y}$ as [39] 


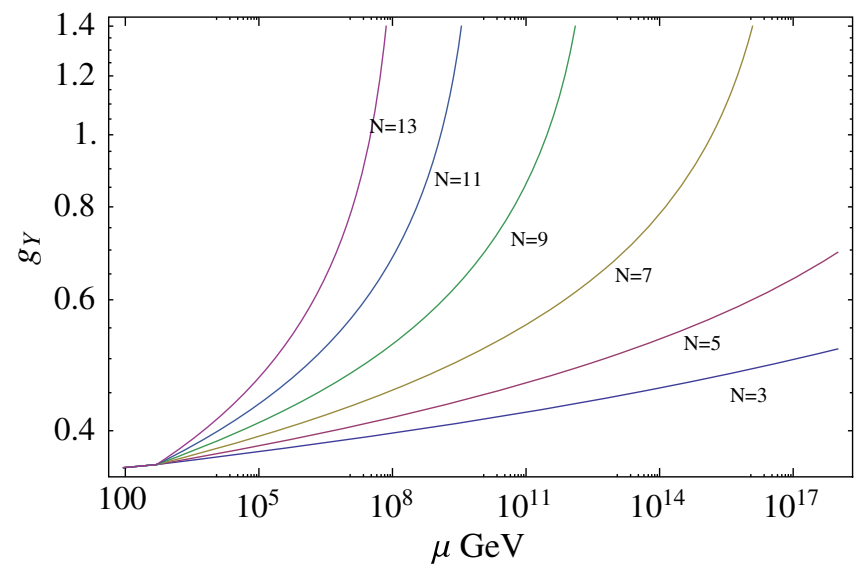

FIG. 2. The running of $g_{Y}$ in terms of a reference energy of $\mu$, depending on each of $N=3,5,7,9,11,13$.

$$
\begin{aligned}
\frac{1}{g_{Y}^{2}(\mu)}= & \frac{1}{g_{Y}^{2}\left(m_{\mathrm{in}}\right)}-\frac{b_{Y}^{\mathrm{SM}}}{(4 \pi)^{2}} \ln \left[\frac{\mu^{2}}{m_{\mathrm{in}}^{2}}\right] \\
& -\theta\left(\mu-m_{\mathrm{thres}}\right) \frac{\left(\Delta b_{Y}^{f}+\Delta b_{Y}^{b}\right)}{(4 \pi)^{2}} \ln \left[\frac{\mu^{2}}{m_{\mathrm{thres}}^{2}}\right],
\end{aligned}
$$

where $\mu$ is a reference energy scale, and we assume that $m_{\text {in }}\left(=m_{Z}\right)<m_{\text {thres }}=500 \mathrm{GeV}$, where $m_{\text {in }} m_{\text {thres }}$ are the initial and threshold mass, respectively. The resulting running of $g_{Y}(\mu)$ versus the scale $\mu$ is shown in Fig. 2 for each of $N=3,5,7,9,11,13$.

\section{MUON $g-2$ AND THE PHYSICS OF EACH $N$}

In this section, we estimate the muon $g-2$ taking into account constraints from LFVs and $Z$ decays and discuss the constraints and prospects for collider physics in some number of $N$.

In addition to the Yukawa interaction explaining the muon $g-2$, we need extra particles and/or interactions to make exotic particles decay into SM ones. Here we summarize the extensions for the cases of $N=3, N=5$, and $N=7$ as follows. ${ }^{3}$

(1) $N=3$ : In this case, we have the interaction term

$$
\mathcal{L}_{\mathrm{ex} 1}=g_{i j} \bar{L}_{L_{i}}^{c} L_{L_{j}} h^{+}+\text {H.c. }
$$

without introducing an extra particle. Then all exotic particles can eventually decay into the SM particles.

(2) $N=5:$ In this case, we have the interaction term

$$
\mathcal{L}_{\mathrm{ex} 2}=g_{i j}^{\prime} \bar{e}_{R_{i}}^{c} e_{R_{j}} h^{++}+\text {H.c. }
$$

\footnotetext{
${ }^{3}$ For $N$ more than 7, model is rather complicated because some fields have to be introduced in order to make new fields into the SM. Thus, we consider $N=3,5,7$.
}

without introducing an extra particle, and exotic particles can decay into the SM particles as in the $N=3$ case. Here it is also worthwhile mentioning that we can explain the active neutrino sector at two-loop level if both extra terms are introduced with an extra doubly (singly) charged particle for the $N=3(5)$ cases. This is called the Zee-Babu model $[40,41]$.

(3) $N=7$ : In this case, we need to introduce $h^{ \pm}$and $h^{ \pm \pm}$in addition to $h^{ \pm \pm \pm}$in order to make it decay into the SM particles. We then have interactions $\mathcal{L}_{\text {ex1(2) }}$ and a new interaction in the scalar potential:

$$
V_{\mathrm{ex}}=\mu_{X} h^{+++} h^{--} h^{-}+\text {c.c., }
$$

with which a triply charged scalar can decay into the SM particles through doubly and singly charged scalar decay by $\mathcal{L}_{\operatorname{ex1}(2)}$ interaction. Note that new Yukawa interactions affect the LFVs, muon $g-2$, and $Z$ decays. In particular, these terms contribute to the muon $g-2$ negatively. Therefore, we require that these terms be small enough to satisfy the sizable muon $g-2$.

\section{A. Muon $g-2$ and flavor constraints for each case}

In this subsection, we scan the Yukawa coupling in Eq. (2.1) and estimate muon $g-2$ taking into account constraints from LFV charged-lepton decay as well as $Z \rightarrow$ $\ell_{i}^{+} \ell_{j}^{-}$processes discussed in the previous section. Here we universally scan $f_{i 1}$ in the range of

$$
f_{i 1} \in\left[10^{-6}, \sqrt{4 \pi}\right],
$$

where the upper bound is the requirement from perturbativity. First, we take a wide mass range of $\left\{m_{\psi}, m_{h}\right\} \in$ $[100,5000] \mathrm{GeV}$ in our parameter scan where $m_{\psi}$ and $m_{h}$ are, respectively, the mass of $L^{\prime}$ and $h^{n}$. In Fig. 3, we show the value of the muon $g-2$ as a function of the exotic lepton mass for $N=3, N=5$, and $N=7$ where the red, green, yellow, and blue color points, respectively, correspond to those with $\ell_{i} \rightarrow \ell_{j} \gamma$ constraints, $\ell_{i} \rightarrow \ell_{j} \gamma$ plus $Z \rightarrow \nu_{i} \bar{\nu}_{j}, \ell_{i} \rightarrow \ell_{j} \gamma$ plus $Z \rightarrow \mu \bar{\mu}$, and $\ell_{i} \rightarrow \ell_{j} \gamma$ plus all of the $Z \rightarrow f_{i} \bar{f}_{j}$. We see that the $Z \rightarrow \mu \bar{\mu}$ and $Z \rightarrow \nu_{i} \bar{\nu}_{j}$ constraints severely exclude the parameter region, and exotic particle masses are preferred to be relatively light as $m_{\psi, h} \lesssim 500 \mathrm{GeV}$. Then we focus on the light mass region which can accommodate the muon $g-2$. The left and right plots in Fig. 4 show the value of the muon $g-2$ as a function of the $L^{\prime}$ and $h^{n}$ masses, respectively, imposing all the constraints as discussed in Fig. 3, where black and pink points, respectively, correspond to the cases of $N=5$ and $N=7$. Furthermore, in Fig. 5 we show a contour plot for $\Delta a_{\mu}$ and $\Delta \mathrm{BR}_{\mu \mu} \equiv \Delta \mathrm{BR}\left(Z \rightarrow \mu^{+} \mu^{-}\right)$on the $\left\{M\left(=m_{h}=\right.\right.$ $\left.\left.m_{\psi}\right), f_{21}\right\}$ plane where we take only $f_{21}$ to be nonzero and 

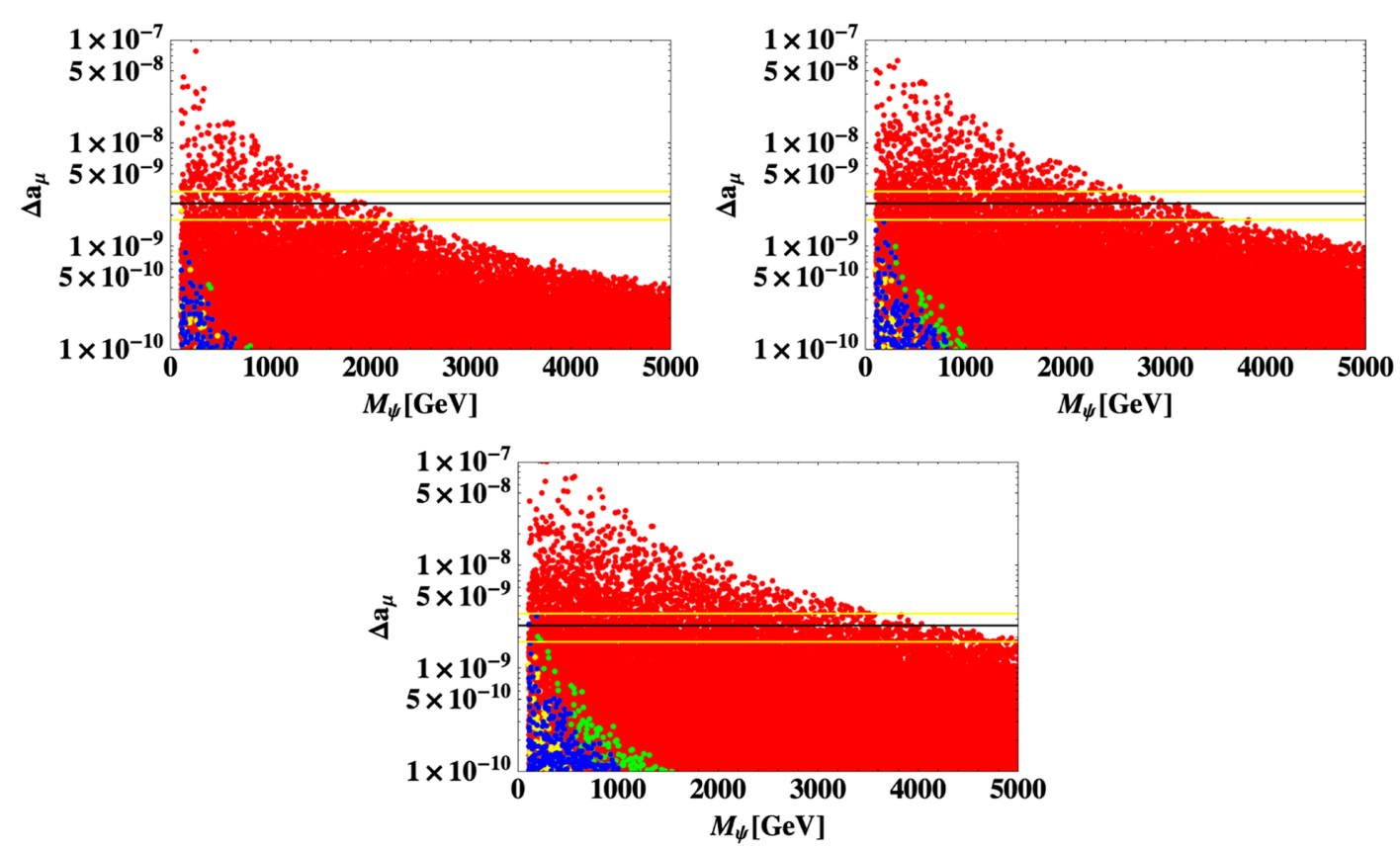

FIG. 3. Muon $g-2$ as a function of $L^{\prime}$ mass obtained from parameter scan for $N=3, N=5$, and $N=7$ where the red, green, yellow, and blue color points, respectively, correspond to those with $\ell_{i} \rightarrow \ell_{j} \gamma$ constraints, $\ell_{i} \rightarrow \ell_{j} \gamma$ plus $Z \rightarrow \nu_{i} \bar{\nu}_{j}, \ell_{i} \rightarrow \ell_{j} \gamma$ plus $Z \rightarrow \mu \bar{\mu}$, and $\ell_{i} \rightarrow \ell_{j} \gamma$ plus all of the $Z \rightarrow f_{i} \bar{f}_{j}$.
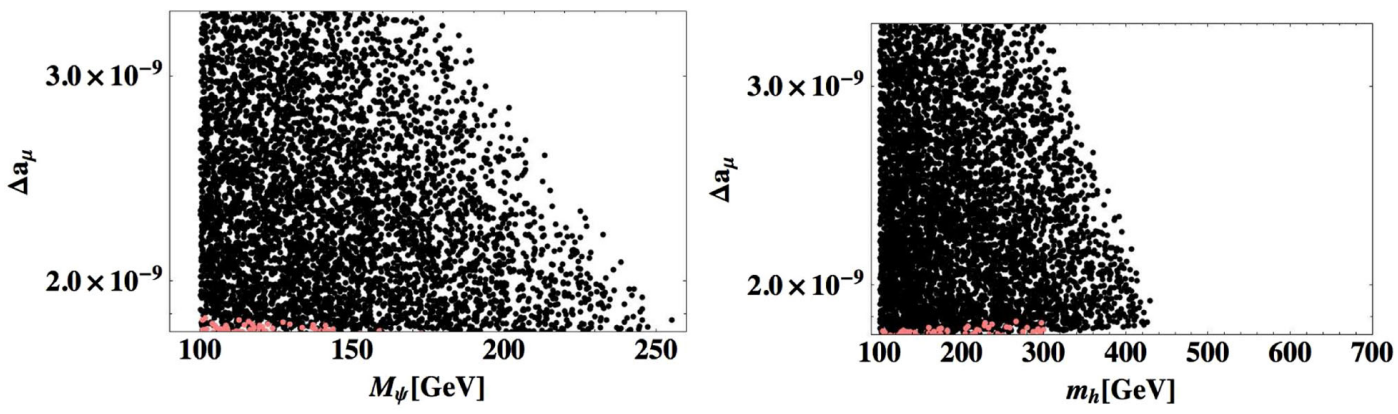

FIG. 4. Muon $g-2$ as a function of $L^{\prime}$ and $h^{n}$ masses (left and right plots) obtained from a parameter scan imposing all the constraints as discussed in Fig. 3, where the black and pink points, respectively, correspond to the cases of $N=5$ and $N=7$. Note here that there are not any allowed points for $N=3$.

other $f_{i j}$ to be zero. In the plots, the (light) yellow region is the $(2 \sigma) 1 \sigma$ region for muon $g-2$, and the shaded region is excluded by $\Delta \mathrm{BR}\left(Z \rightarrow \mu^{+} \mu^{-}\right)$. Thus, one find that the mass scale is constrained by $\Delta \mathrm{BR}_{\mu \mu}$ even if only $f_{21}$ is nonzero. We thus find that the $L^{\prime}$ mass should be relatively light as $m_{\psi} \sim(150,200,250) \mathrm{GeV}$ for $N=(3,5,7)$ to explain the muon $g-2$ within $1 \sigma$, while the charged scalar mass $m_{h}$ can be heavier than $m_{\psi}$.

\section{B. Collider physics and constraints}

In explaining the muon $g-2$ by the interaction Eq. (2.1), the mass scale of exotic lepton doublet $L^{\prime}$ is required to be less than $\sim 300 \mathrm{GeV}$. Thus, the exotic charged lepton can be produced at the LHC with a sizable production cross section, and we should take into account collider constraints to check if the mass scale for explaining the muon $g-2$ is allowed. In our study, we focus on the exotic charged lepton with the highest electric charge since it has the largest pair production cross section and provide the most stringent constraint.

First, we estimate the pair production cross section of the highest charged leptons for each case. These charged leptons can be pair produced by the Drell-Yan (DY) process $q \bar{q} \rightarrow Z / \gamma \rightarrow \psi^{+n} \psi^{-n}$ and also by the photon fusion (PF) process $\gamma \gamma \rightarrow \psi^{+n} \psi^{-n}$ [42-44]. Here we estimate the cross section by applying MADGRAPH/MADEVENT5 [45], where the necessary Feynman rules and relevant parameters of the model are implemented using FeynRules 2.0 [46], and the NNPDF2 3LO1 parton distribution function [47] is adopted. In Fig. 6, we show the cross sections including both DY and PF processes at the LHC 8 (13) $\mathrm{TeV}$ for the left (right) 

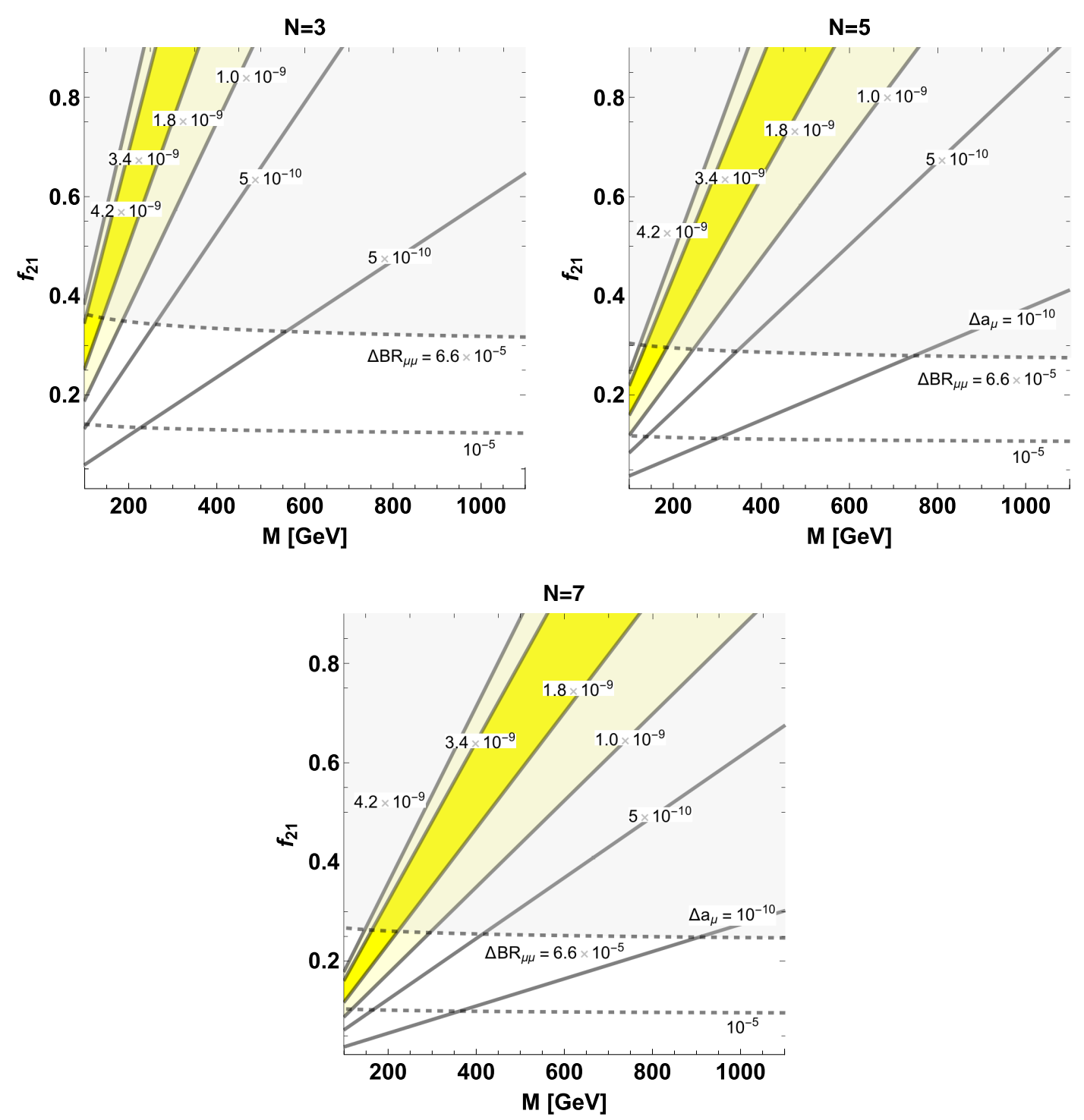

FIG. 5. Contours of $\Delta a_{\mu}$ and $\Delta \mathrm{BR}_{\mu \mu} \equiv \Delta \mathrm{BR}\left(Z \rightarrow \mu^{+} \mu^{-}\right)$on the $\left\{M\left(=m_{h}=m_{\psi}\right), f_{21}\right\}$ plane where we take only $f_{21}$ to be nonzero and the other $f_{i j}$ to be zero. The (light) yellow region is the $(2 \sigma) 1 \sigma$ region for muon $g-2$ and the shaded region is excluded by $\Delta \mathrm{BR}\left(Z \rightarrow \mu^{+} \mu^{-}\right)$.

plots. We thus find that the cross section is large when electric charge is increased, where the PF process highly enhances the cross section.

Second, we list the decay chain of the highest charged lepton for each case.

(1) $N=3$ : The decay chain of $E^{ \pm \pm}$is

$$
\psi^{ \pm \pm} \rightarrow \ell_{i}^{ \pm} h^{ \pm(*)} \rightarrow \ell_{i}^{ \pm} \ell_{j}^{ \pm} \nu,
$$

where the charged scalar can be either on shell or off shell. Thus, the $\psi^{++} \psi^{--}$pair production process gives four charged leptons with missing transverse energy. The singly charged scalar with $m_{h^{+}}>$ $100 \mathrm{GeV}$ is allowed by the collider experiment, and we require that the mass be heavier than $100 \mathrm{GeV}$ [34].
(2) $N=5$ : The decay chain of $\psi^{ \pm \pm \pm}$is

$$
\begin{aligned}
\psi^{ \pm \pm \pm} & \rightarrow \ell_{i}^{ \pm} h^{ \pm \pm(*)} \\
& \rightarrow \ell_{i}^{ \pm} \ell_{j}^{ \pm} \ell_{k}^{ \pm}\left[\rightarrow \ell_{i}^{ \pm} h^{ \pm} h^{ \pm} \rightarrow \ell_{i}^{ \pm} \ell_{j}^{ \pm} \ell_{k}^{ \pm} \nu\right],
\end{aligned}
$$

where the charged scalar can be either on shell or off shell as the previous case, and the process in square brackets can be induced by introducing a singly charged scalar with interaction Eq. (3.1). Thus, the $\psi^{+++} \psi^{---}$pair production process gives six charged leptons. Note that the doubly charged scalar mass is constrained by the LHC data as $m_{h^{ \pm \pm}} \gtrsim 700$ $800 \mathrm{GeV}$ and $m_{h^{ \pm \pm}} \gtrsim 400 \mathrm{GeV}$ when the $h^{ \pm \pm}$decays into $e^{ \pm} e^{ \pm}\left(\mu^{ \pm} \mu^{ \pm}\right)$and $\tau^{ \pm} \tau^{ \pm}$, respectively [48,49]. The constraint is looser as $m_{h^{ \pm \pm}} \gtrsim 200 \mathrm{GeV}$ when 

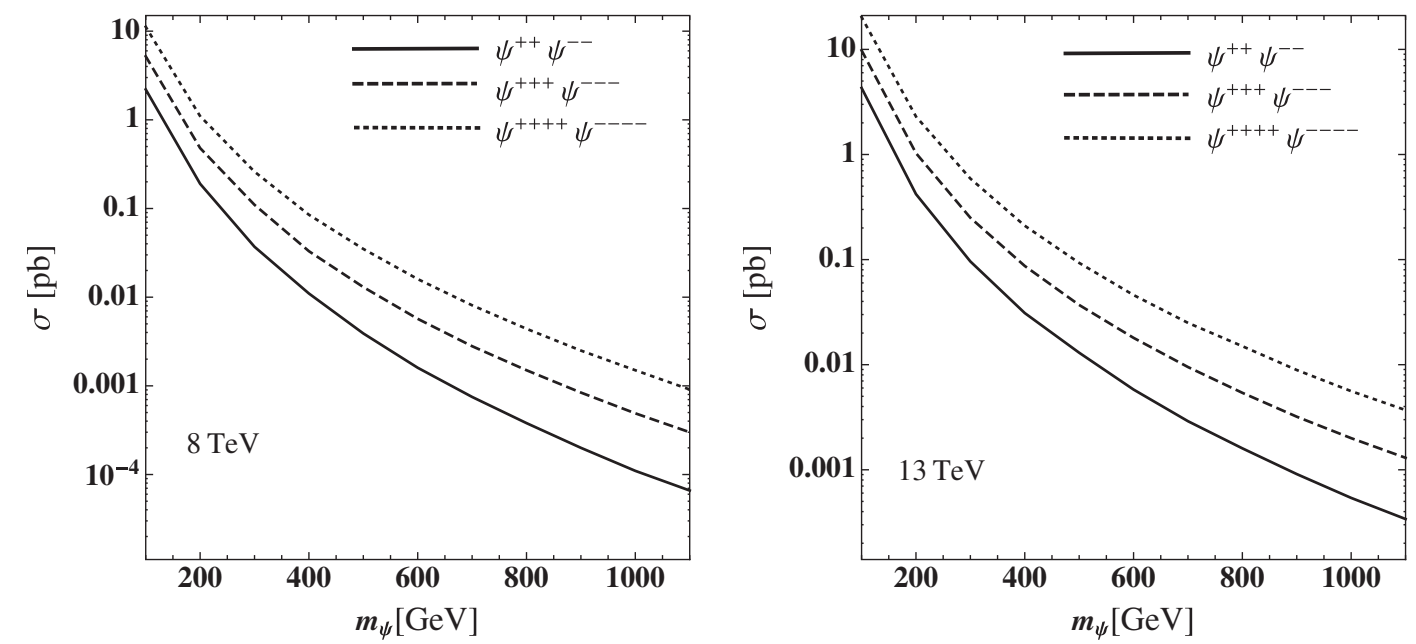

FIG. 6. The pair production cross section of the exotic charged leptons with the highest electric charge for each case at the LHC 8 (13) TeV for the left (right) plots.

$h^{ \pm \pm}$dominantly decays via the $h^{ \pm \pm} \rightarrow h^{ \pm} h^{ \pm} \rightarrow$ $\ell_{i}^{ \pm} \ell_{j}^{ \pm} \nu \nu$ process. To explain the muon $g-2$, we require $h^{ \pm \pm}$to dominantly decay into singly charged scalars [50].

(3) $N=7$ : The decay chain of $\psi^{ \pm \pm \pm \pm}$is

$$
\begin{aligned}
\psi^{ \pm \pm \pm \pm} & \rightarrow \ell_{i}^{ \pm} h^{ \pm \pm \pm(*)} \rightarrow \ell_{i}^{ \pm} h^{ \pm \pm(*)} h^{ \pm(*)} \\
& \rightarrow h^{ \pm(*)} h^{ \pm(*)} h^{ \pm(*)} \rightarrow \ell_{i}^{ \pm} \ell_{j}^{ \pm} \ell_{k}^{ \pm} \ell_{l}^{ \pm} \nu \nu \nu,
\end{aligned}
$$

where the triply charged scalar decays via interaction in Eq. (3.3). Also, as in the previous case, we require doubly charged scalar decay into a same-sign singly charged scalar pair. Thus, the $\psi^{++++} \psi^{----}$pair production process gives eight charged leptons with missing transverse energy. In general, the constraint on the mass of the triply charged scalar is weaker than that on $\psi^{ \pm \pm \pm \pm}$, and we will not explicitly discuss the constraint.

Finally, let us discuss collider constraints on our scenario to explain the muon $g-2$. We note that the highest charged lepton dominantly decays into $\psi^{ \pm n} \rightarrow \mu^{ \pm} h^{ \pm n-1}$ since the $f_{21}$ coupling is required to be large to explain the muon $g-2$. In addition to the conditions discussed above, we classify benchmark scenarios as follows:

(a) The singly charged scalar decay into $\ell=e, \mu$ in the decay chain and exotic charged lepton has sufficiently short decay length.

(b) Exotic charged leptons have long decay length and pass through the detector.

(c) The singly charged scalar decays into the $\tau \nu$ mode, and the highest charged scalar mass is slightly lighter than that of the highest charged lepton.

For scenario (a), an inclusive multilepton search constrains the cross section where the upper bound of the cross section is $\sim 1 \mathrm{fb}$ at the LHC $8 \mathrm{TeV}$ for the signal in which the number of charged leptons $N_{\ell}(\ell=e, \mu)$ is
$N_{\ell} \geq 3$ [51]. Comparing the cross section for $8 \mathrm{TeV}$ in Fig. 6, the charged-lepton masses are required to be $m_{\psi} \gtrsim(650,900,1100) \mathrm{GeV}$. In this scenario, the region explaining the muon $g-2$ in $1 \sigma$ is excluded for all $N$, and the largest value of the muon $g-2$ is roughly $\Delta a_{\mu} \sim 10^{-10}$ for each case. Scenario (b) can be realized when the charged scalar in the decay chains is off shell and the extra couplings in Eqs. (3.1)-(3.3) are sufficiently small. For the long-lived charged particle, the upper bound of the cross section is given in Ref. [52] for the LHC $13 \mathrm{TeV}$. Comparing the result for the chargino, we find the upper

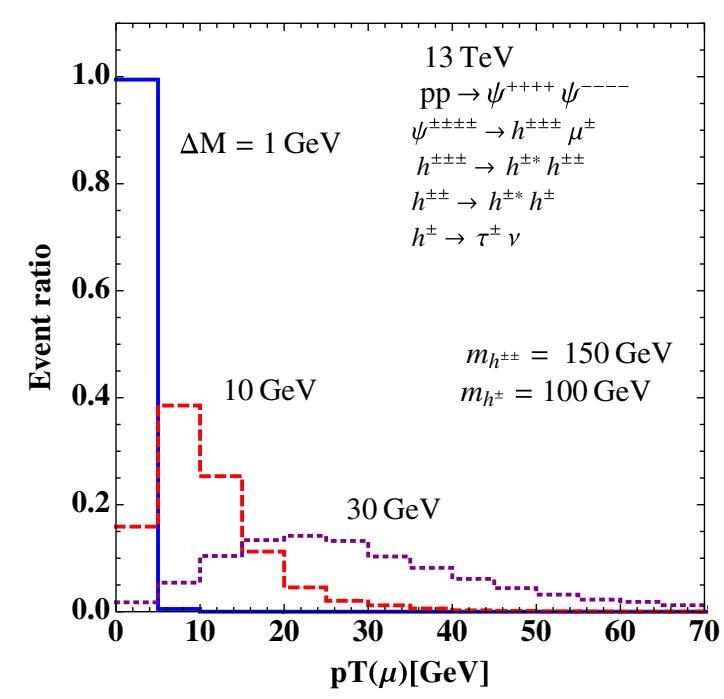

FIG. 7. The distribution of the transverse momentum of muon $\mu$ in $\psi^{ \pm \pm \pm \pm} \rightarrow h^{ \pm \pm \pm} \mu^{ \pm} \rightarrow h^{ \pm \pm} \mu^{ \pm} \tau^{ \pm} \nu \rightarrow h^{ \pm} \mu^{ \pm} \tau^{ \pm} \tau^{ \pm} \nu \nu \rightarrow$ $\mu^{ \pm} \tau^{ \pm} \tau^{ \pm} \tau^{ \pm} \nu \nu \nu$ decay at the LHC $13 \mathrm{TeV}$ (neutrino and antineutrino are not distinguished here); the vertical axis shows the event ratio defined by $N_{\text {bin }} / N_{\text {total }}$ where $N_{\text {total }}$ and $N_{\text {bin }}$ indicate number of events in total and those inside the corresponding bins. Here $\Delta M$ indicates the mass difference between $\psi^{ \pm \pm \pm \pm}$and $h^{ \pm \pm \pm}$. 

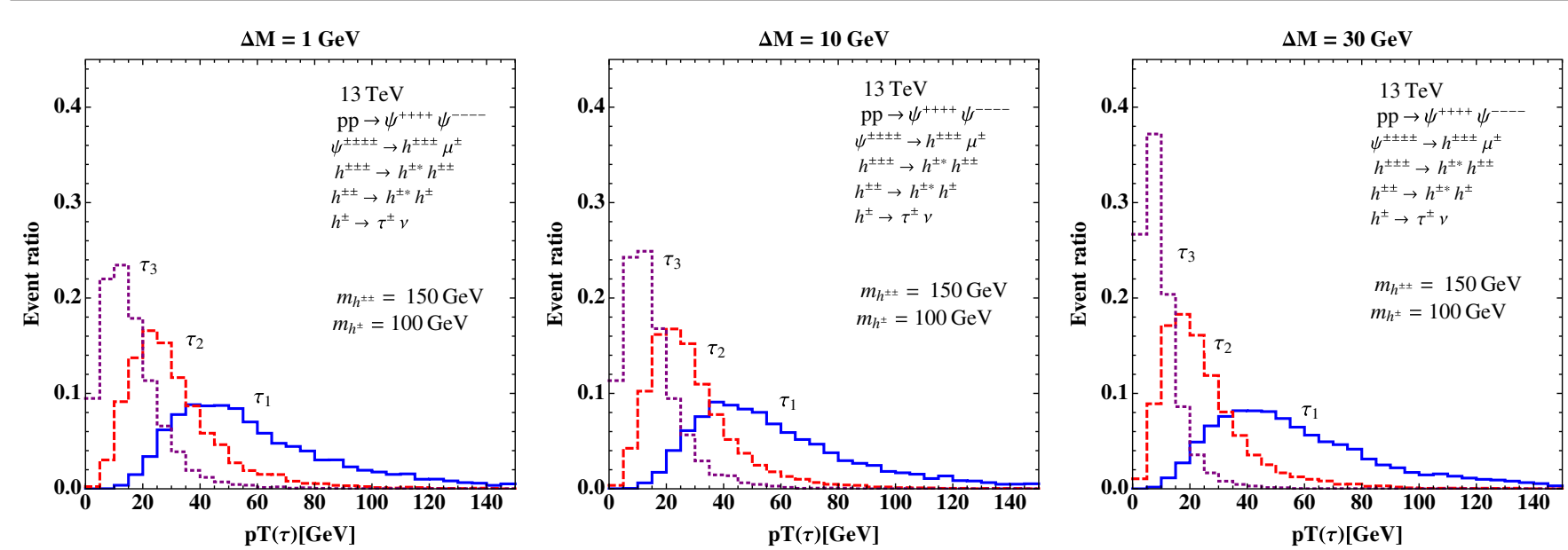

FIG. 8. The distribution of the transverse momentum of muon $\tau$ leptons for the same process as Fig. 7 where three $\tau$ leptons are distinguished by the transverse momentum as $p_{T}\left(\tau_{3}\right)<p_{T}\left(\tau_{2}\right)<p_{T}\left(\tau_{1}\right)$ for each event.

limit is less than $1 \mathrm{fb}$, and since we have multiple charged leptons, the constraint will be stronger. Thus, the collider constraint in this scenario is stronger than scenario (a), and we cannot expect a sizable muon $g-2$. For scenario (c), the decay chain provides a signature for each case such that case (1) gives a low energy muon with missing transverse energy, and cases (2) and (3) give a multitau lepton signature with a low energy muon since we require that the mass difference between $\psi^{-n-1}$ and $h^{ \pm n}$ be small and $h^{ \pm n}$ be on shell. In Fig. 7, we show the event ratio for the distribution of the transverse momentum of muon $\mu$ in the $\psi^{ \pm \pm \pm \pm} \rightarrow h^{ \pm \pm \pm} \mu^{ \pm} \rightarrow h^{ \pm \pm} \mu^{ \pm} \tau^{ \pm} \nu \rightarrow h^{ \pm} \mu^{ \pm} \tau^{ \pm} \tau^{ \pm} \nu \nu \rightarrow$ $\mu^{ \pm} \tau^{ \pm} \tau^{ \pm} \tau^{ \pm} \nu \nu \nu$ decay chain at the LHC $13 \mathrm{TeV}$ for different values of $\Delta M$ indicating a mass difference between $\psi^{ \pm \pm \pm \pm}$ and $h^{ \pm \pm \pm}$where the behaviors are similar if we change colliding energy from 13 to 8 or $14 \mathrm{TeV}$; here we consider case (3), but we will have similar results for the other cases. The masses of $h^{ \pm \pm}$and $h^{ \pm}$are also fixed to be $m_{h^{ \pm \pm}}=$ $150 \mathrm{GeV}$ and $m_{h^{ \pm}}=100 \mathrm{GeV}$. In Fig. 8, we also show the event ratios for the distribution of transverse momentum of $\tau$ in the same process, where three $\tau$ leptons are distinguished by transverse momentum as $p_{T}\left(\tau_{3}\right)<p_{T}\left(\tau_{2}\right)<$ $p_{T}\left(\tau_{1}\right)$ for each event. It is found that the transverse momentum of some $\tau$ leptons is generally sizable, and they can be detected at the detector. On the other hand, the transverse momentum of $\mu$ tends to be small for $\Delta M \lesssim 10 \mathrm{GeV}$, and it will be missed by an event trigger. For the multilepton search in Ref. [51], they require that one muon or electron should have $p_{T}>26 \mathrm{GeV}$ and $p_{T}>$ $15 \mathrm{GeV}$ from the second muon (electron). As a result, the number of dimuon signal events becomes less than $\sim 0.1 \%$ after the trigger for $\Delta M=10 \mathrm{GeV}$. We thus see that if $\Delta M \lesssim 10 \mathrm{GeV}$, most events are missed by the event trigger, and we can escape the experimental bound. Therefore, scenario (c) with small $\Delta M$ can still be allowed since an analysis of the multitau signature is more difficult and the explicit bound is not given. Thus, we conclude that to obtain a sizable muon $g-2$ by interaction Eq. (2.1), we should rely on this specific scenario. Therefore, the multitau signature is important to test the mechanism to explain the muon $g-2$, although an analysis of it is challenging.

\section{CONCLUSIONS}

We have analyzed the muon $g-2$, LFVs, and $Z$ decays including collider physics in multicharged particles. We have found that LFVs do not restrict the allowed region of the muon $g-2$, while the $Z \rightarrow \nu_{i} \bar{\nu}_{j}$ invisible decay and $Z \rightarrow \mu \bar{\mu}$ give stringent constraints, and the allowed region drastically disappears. Also, larger $N$ increases the allowed region of the muon $g-2$. However, once we consider the constraint of collider physics, the typical size of the muon $g-2$ is of the order $10^{-10}$, depending on the benchmark scenarios (a)-(c). To obtain a sizable muon $g-2$ of $\mathcal{O}\left(10^{-9}\right)$, we have found that a specific scenario is required for the decay chain of the charged particles in which the mass of $L^{\prime}$ is slightly heavier than $h^{ \pm n}$ and charged scalar bosons decay into a mode only including $\tau$ and neutrinos. Therefore, an analysis of the multitau lepton signature is important to fully test the scenario to explain the muon $g-2$.

\section{ACKNOWLEDGMENTS}

This research was supported by an appointment to the JRG Program at the APCTP through the Science and Technology Promotion Fund and Lottery Fund of the Korean Government. This work was also supported by the Korean Local Governments, Gyeongsangbuk-do Province and Pohang City (H.O.). H. O. is sincerely grateful to KIAS and all its members. 
[1] K. Hagiwara, R. Liao, A. D. Martin, D. Nomura, and T. Teubner, J. Phys. G 38, 085003 (2011).

[2] A. Keshavarzi, D. Nomura, and T. Teubner, Phys. Rev. D 97, 114025 (2018).

[3] J. Grange et al. (Muon g-2 Collaboration), arXiv:1501.06858.

[4] H. Iinuma (J-PARC Muon g-2/EDM Collaboration), J. Phys. Conf. Ser. 295, 012032 (2011).

[5] W. Altmannshofer, S. Gori, M. Pospelov, and I. Yavin, Phys. Rev. Lett. 113, 091801 (2014).

[6] G. Mohlabeng, Phys. Rev. D 99, 115001 (2019).

[7] W. Abdallah, A. Awad, S. Khalil, and H. Okada, Eur. Phys. J. C 72, 2108 (2012).

[8] M. Lindner, M. Platscher, and F. S. Queiroz, Phys. Rep. 731, 1 (2018).

[9] S. M. Barr and A. Zee, Phys. Rev. Lett. 65, 21 (1990); 65, 2920(E) (1990).

[10] A. E. Cárcamo Hernández, S. Kovalenko, R. Pasechnik, and I. Schmidt, Eur. Phys. J. C 79, 610 (2019).

[11] E. Ma and M. Raidal, Phys. Rev. Lett. 87, 011802 (2001); 87, 159901(E) (2001).

[12] H. Okada and K. Yagyu, Phys. Rev. D 89, 053008 (2014).

[13] S. Baek, H. Okada, and T. Toma, Phys. Lett. B 732, 85 (2014).

[14] H. Okada and K. Yagyu, Phys. Rev. D 90, 035019 (2014).

[15] H. Okada, T. Toma, and K. Yagyu, Phys. Rev. D 90, 095005 (2014).

[16] H. Okada and K. Yagyu, Phys. Rev. D 93, 013004 (2016).

[17] H. Okada and K. Yagyu, Phys. Lett. B 756, 337 (2016).

[18] T. Nomura and H. Okada, Phys. Lett. B 756, 295 (2016).

[19] P. Ko, T. Nomura, H. Okada, and Y. Orikasa, Phys. Rev. D 94, 013009 (2016).

[20] S. Baek, T. Nomura, and H. Okada, Phys. Lett. B 759, 91 (2016).

[21] T. Nomura and H. Okada, Phys. Rev. D 94, 075021 (2016).

[22] S. Lee, T. Nomura, and H. Okada, Nucl. Phys. B931, 179 (2018).

[23] C. W. Chiang, H. Okada, and E. Senaha, Phys. Rev. D 96, 015002 (2017).

[24] A. Das, T. Nomura, H. Okada, and S. Roy, Phys. Rev. D 96, 075001 (2017).

[25] T. Nomura and H. Okada, Phys. Rev. D 96, 015016 (2017).

[26] T. Nomura and H. Okada, Int. J. Mod. Phys. A 33, 1850089 (2018).

[27] K. Cheung and H. Okada, Phys. Lett. B 774, 446 (2017).

[28] K. Cheung and H. Okada, Phys. Rev. D 97, 075027 (2018).

[29] Y. Cai, J. Herrero-García, M. A. Schmidt, A. Vicente, and R. R. Volkas, Front. Phys. 5, 63 (2017).
[30] T. Nomura and H. Okada, Phys. Dark Universe 21, 90 (2018).

[31] S. Baumholzer, V. Brdar, and P. Schwaller, J. High Energy Phys. 08 (2018) 067.

[32] N. Chakrabarty, C. W. Chiang, T. Ohata, and K. Tsumura, J. High Energy Phys. 12 (2018) 104.

[33] C. H. Chen and T. Nomura, Phys. Rev. D 100, 015024 (2019).

[34] M. Tanabashi et al. (Particle Data Group), Phys. Rev. D 98, 030001 (2018).

[35] A. M. Baldini et al. (MEG Collaboration), Eur. Phys. J. C 76, 434 (2016).

[36] F. Renga (MEG Collaboration), Hyperfine Interact. 239, 58 (2018).

[37] CEPC-SPPC Study Group, Reports No. IHEP-CEPC-DR2015-01, No. IHEP-TH-2015-01, and No. IHEP-EP-2015$01,2015$.

[38] B. Aubert et al. (BABAR Collaboration), Phys. Rev. Lett. 104, 021802 (2010).

[39] S. Kanemura, K. Nishiwaki, H. Okada, Y. Orikasa, S. C. Park, and R. Watanabe, Prog. Theor. Exp. Phys. 2016, 123B04 (2016).

[40] A. Zee, Phys. Lett. 93B, 389 (1980); 95B, 461(E) (1980).

[41] K. S. Babu, Phys. Lett. B 203, 132 (1988).

[42] K. S. Babu and S. Jana, Phys. Rev. D 95, 055020 (2017).

[43] K. Ghosh, S. Jana, and S. Nandi, J. High Energy Phys. 03 (2018) 180.

[44] T. Ghosh, S. Jana, and S. Nandi, Phys. Rev. D 97, 115037 (2018).

[45] J. Alwall, R. Frederix, S. Frixione, V. Hirschi, F. Maltoni, O. Mattelaer, H.-S. Shao, T. Stelzer, P. Torrielli, and M. Zaro, J. High Energy Phys. 07 (2014) 079.

[46] A. Alloul, N. D. Christensen, C. Degrande, C. Duhr, and B. Fuks, Comput. Phys. Commun. 185, 2250 (2014).

[47] C. S. Deans (NNPDF Collaboration), arXiv:1304.2781.

[48] CMS Collaboration, CERN Report No. CMS-PAS-HIG-16036, 2017.

[49] M. Aaboud et al. (ATLAS Collaboration), Eur. Phys. J. C 78, 199 (2018).

[50] R. Primulando, J. Julio, and P. Uttayarat, J. High Energy Phys. 08 (2019) 024.

[51] G. Aad et al. (ATLAS Collaboration), J. High Energy Phys. 08 (2015) 138.

[52] M. Aaboud et al. (ATLAS Collaboration), Phys. Rev. D 99, 092007 (2019). 